\title{
Single-Incision Laparoscopic Sterilization of the Cheetah (Acinonyx jubatus)
}

\author{
Marthinus J. Hartman ${ }^{1}$, MMedVet(Surg), Eric Monnet ${ }^{2}$, PhD, Diplomate ACVS, ECVS, \\ Robert M. Kirberger ${ }^{1}$, DVSc, Diplomate ECVDI, Anne Schmidt-Küntzel ${ }^{3}$, PhD, Martin L. Schulman ${ }^{4}$, \\ MMedVet (Gyn), Jana A. Stander ${ }^{1}$, DipVetNur, George F. Stegmann ${ }^{1}$, Diplomate ECVA, \\ and Johan P. Schoeman ${ }^{1}$, PhD, Diplomate, ECVIM
}

\footnotetext{
${ }^{1}$ Department of Companion Animal Clinical Studies, Faculty of Veterinary Science, University of Pretoria, Pretoria, South Africa, ${ }^{2}$ Department of Clinical Sciences, Colorado State University, Colorado, ${ }^{3}$ Cheetah Conservation Fund, Otjiwarongo, Namibia and ${ }^{4}$ Department of Production Animal Studies, Faculty of Veterinary Science, University of Pretoria, Pretoria, South Africa
}

Corresponding Author

Marthinus Hartman, Department of Companion Animal Clinical Studies, Faculty of Veterinary Science, University of Pretoria, Soutpan Road, Onderstepoort Veterinary Academic Hospital, Room 4-20, Onderstepoort, 0110, South Africa.

E-mail: marthinus.hartman@up.ac.za

Objective: To describe laparoscopic ovariectomy and salpingectomy in the cheetah (Acinonyx jubatus) using single-incision laparoscopic surgery (SILS).

Study Design: Prospective cohort.

Animals: Female cheetahs (Acinonyx jubatus) $(\mathrm{n}=21)$.

Methods: Cheetahs were randomly divided to receive either ovariectomy $(\mathrm{n}=11)$ or salpingectomy $(\mathrm{n}=10)$. The use and complications of a SILS port was evaluated in all of cheetahs. Surgery duration and insufflation volumes of carbon dioxide $\left(\mathrm{CO}_{2}\right)$ were recorded and compared across procedures.

Results: Laparoscopic ovariectomy and salpingectomy were performed without complications using a SILS port. The poorly-developed mesosalpinx and ovarian bursa facilitated access to the uterine tube for salpingectomy in the cheetah. The median surgery duration for ovariectomy was 24 minutes (interquartile range 3) and for salpingectomy was 19.5 minutes (interquartile range 3$)(P=.005)$. The median volume of $\mathrm{CO}_{2}$ used for ovariectomy was $11.25 \mathrm{~L}$ (interquartile range $3.08)$ and for salpingectomy was $4.90 \mathrm{~L}$ (interquartile range 2.52$),(P=.001)$

Conclusions: Laparoscopic ovariectomy and salpingectomy can be performed in the cheetah using SILS without perioperative complications. Salpingectomy is faster than ovariectomy and requires less total $\mathrm{CO}_{2}$ for insufflation.

There are an estimated 1,600 captive cheetahs (Acinonyx jubatus) worldwide. ${ }^{1}$ Recent amendments to legislation in Namibia require permanent sterilization of captive female predators, including cheetahs. ${ }^{2}$ Various methods of population control have been used in captive cheetah populations, including separation of sexes and induction of contraception by down-regulation of LH and FSH secretion using the GnRH analogue deslorelin (Suprelorin ${ }^{\mathbb{B}}$ Virbac Pty Ltd, Halfway House, South Africa). ${ }^{3,4}$ Use of deslorelin does require the cheetah to be immobilized and be implanted every 12 months for maintenance of contraception, ${ }^{3}$ and the ongoing need for repeated procedures can be prohibitive in terms of cost and management efforts. The requirement for sterilization of an endangered species does remain controversial.

Ovariectomy is a safe method of sterilization in domestic animals $^{5-8}$ and is easily performed via laparoscopic surgery. Other laparoscopic sterilization techniques have been reported including ovariohysterectomy, uterine horn occlusion, and laparoscopic-assisted ovariohysterectomy. ${ }^{9-13}$ Laparoscopic ovariectomy has been performed in domestic cats using laser and bipolar electrocoagulation, ${ }^{14}$ and in 1 cheetah after removal of an abdominal foreign body. ${ }^{15}$ Laparoscopic ovariectomy, salpingectomy, ovariohysterectomy, and hysterectomy using multiple ports have been reported in the lion, ${ }^{16-}$ 18 and ovariectomy using single-incision laparoscopic surgery (SILS) has been performed in 2 tigers. ${ }^{19}$ Laparoscopic sterilization of the cheetah has not been reported.

In countries where permanent sterilization of wild carnivores in captivity is legislated, the use of laparoscopic salpingectomy in a mobile theater could provide a fast and uncomplicated procedure compared to ovariectomy, ovariohysterectomy, or hysterectomy provided that the effects on uterine health are determined to be comparable to other methods of population control. The association of salpingectomy with later uterine pathology in domestic animals or large felids is currently undefined, while in women, salpingectomy does not appear to have any significant adverse effects on uterine health. ${ }^{20}$ The effect of both ovariectomy and salpingectomy on the social behavior in wild African carnivores has not been reported.

Laparoscopic ovariectomy requires either 1,2 , or 3 cannulas in $\operatorname{dog} s^{13,21-24}$ and the lion. ${ }^{18}$ The utilization of a 
SILS port has been described for ovariectomy and other abdominal procedures in $\operatorname{dogs}^{25-27}$ and its effective resterilization and re-use has been reported. ${ }^{28}$ Cited advantages include access to the abdominal cavity without a Veress needle and the ability to place 3 different ports through a single incision.

The aim of this study was to investigate the use and potential complications of SILS associated with ovariectomy and salpingectomy in cheetahs.

\section{MATERIALS AND METHODS}

Twenty-one cheetahs from 2 research conservation centers in northern Namibia (facilities A and B) were included. The study was approved by the Animal Use and Care and Research Committees of the Faculty of Veterinary Science, University of Pretoria (protocol number v014-14). The study was performed under the permit 1919/2014 of the Namibian Ministry of Environment and Tourism.

Cheetahs were randomized to receive either ovariectomy or salpingectomy according to a random number table. Age was obtained from the animal's record and body weight was obtained after anesthetic induction. Packed cell volume and urine specific gravity were measured to confirm adequate hydration and assess kidney function. Body fat distribution was assessed macroscopically during surgery. The total volume of $\mathrm{CO}_{2}$ used for insufflation was recorded. The number of applications of the vessel-sealing device required to complete surgeries and the ability to perform both procedures with regular instruments as opposed to curved and angulated instruments were noted. Any extension of the SILS portal incision for removal of ovaries was recorded. Cheetahs with ovaries that had paraovarian cysts were noted. Intraoperative complications were recorded and the reproductive organs were observed for signs of involuntary contraction.

\section{Anesthesia}

Free access to water was allowed, but food was withheld for approximately 12 hours before surgery. Immobilization was achieved with tiletamine and zolazepam ((Zoletil ${ }^{\mathbb{B}}, 100 \mathrm{mg} / \mathrm{mL}$, Virbac, Halfway House, South Africa) at $1.2 \mathrm{mg} / \mathrm{kg}$ and medetomidine (Domitor ${ }^{\circledR}, 1 \mathrm{mg} / \mathrm{mL}$ Pfizer Animal Health, Sandton, South Africa) at $0.03 \mathrm{mg} / \mathrm{kg}$ intramuscular (IM). At facility $\mathrm{A}$, the drug combinations were delivered by a remote projection system using $3 \mathrm{~mL}$ darts with $30 \mathrm{~mm}$ collared side-port needles (DanInject, Denmark). At facility B, the cheetahs were trained to enter a squeeze cage in which they could be injected by hand. Once immobilized, the cheetah was intubated and anesthesia maintained with isoflurane in oxygen via a circle rebreathing circuit. During abdominal insufflation, positive pressure ventilation was applied with a mechanical ventilator ((Endoflator, Karl Storz GMBH \& Co. KG, Tuttlingen, Germany). Lactated Ringer's solution at $10 \mathrm{~mL} / \mathrm{kg} /$ hour was administered intravenously (IV) until extubation. Morphine at $0.2 \mathrm{mg} / \mathrm{kg}$ subcutaneously (SC) was administered immediately after intubation for intraoperative analgesia and $1.5 \mathrm{~mL}$ of ropivacaine $\left(\right.$ Naropin $^{\circledR}, 7.5 \mathrm{mg} / \mathrm{mL}$, AstraZeneca Pharmaceuticals, Sunninghill Johannesburg, South Africa) was injected SC at the surgical incision site prior to incision. Postoperative analgesia was provided with meloxicam (Metacam $^{\circledR}, 5 \mathrm{mg} / \mathrm{mL}$, Boehringer Ingelheim, Pine Avenue, Randburg, South Africa) at $0.3 \mathrm{mg} / \mathrm{kg} \mathrm{SC}$ prior to anesthetic recovery.

The surgical site was clipped and the cheetah was positioned in dorsal recumbency in an endoscopic positioner (Apexx TT, Englewood, CO) for abdominal ultrasound to assess its reproductive status.

\section{Surgical Procedure}

The ventral abdomen was prepared for aseptic surgery and a sterile, transparent, plastic adhesive surgical drape (Steridrape $1014,320 \mathrm{~cm} \times 214 \mathrm{~cm}, 48 \mathrm{~cm} \times 25 \mathrm{~cm}, 3 \mathrm{M}$, Mexico, St. Paul) was applied to skin surface. Surgery was performed by 2 surgeons (E.M. and M.H.). A $2.5 \mathrm{~cm}$ skin incision was made immediately caudal to the umbilicus after infiltration of local anesthetic. The linea alba was exposed and 2 horizontal stay sutures were placed on either side of the linea alba to facilitate placement of the SILS port (SILS ${ }^{\mathrm{TM}}$ Port, Covidien ${ }^{\mathrm{TM}}$ ). A $2 \mathrm{~cm}$ incision was made in the linea alba and the SILS port was introduced through the incision with curved Spencer-Wells forceps. Two $5 \mathrm{~mm}$ cannulas and a 5-12 $\mathrm{mm}$ cannula were placed in the SILS port. The abdominal cavity was insufflated with $\mathrm{CO}_{2}$ to a pressure of $13 \mathrm{mmHg}$ using an automatic insufflator (Endoflator, Karl Storz GMBH \& Co. KG).

A $5 \mathrm{~mm} 0^{\circ}$ endoscope (HOPKINS Optik, Karl Storz GMBH \& Co. KG) was inserted and the abdominal cavity was inspected.

Ovariectomy. With the cheetah tilted $45^{\circ}$ in a left oblique position, the rigid endoscope was inserted and the suspensory ligament was identified. A $5 \mathrm{~mm}$ atraumatic grasping forceps (Click Line BABCOCK Grasping Forceps, $5 \mathrm{~mm}, 45 \mathrm{~cm}$, Karl Storz GMBH \& Co. KG) was inserted to grasp the proper ligament (Fig 1A). The ovary was elevated ventrally, approximately half way to the abdominal wall, and a $10 \mathrm{~mm}$ vessel sealing device (Atlas, LigaSureTM ValleylabTM Covidien, Manfield, MA, USA) was inserted through the $5-12 \mathrm{~mm}$ portal. The ovarian artery and vein were sealed and transected (Fig 1B) followed by the suspensory ligament (Fig 1C) and uterine horn, close to the proper ligament, which freed the entire ovary (Fig 1D). The tip of the uterine horn and ovarian pedicle were then inspected for hemorrhage. After removal of the rigid endoscope and the vessel sealing device, the SILS port was removed and the ovary was retrieved from the abdominal cavity together with the port. The cheetah was tilted back to the neutral position, the SILS port was replaced, and insufflation to $13 \mathrm{~mm} \mathrm{Hg}$ was re-established. The cheetah was then tilted $45^{\circ}$ to the right oblique position and the left ovariectomy was performed as described above.

Salpingectomy. The cheetah was positioned as for ovariectomy and the rigid endoscope, the $5 \mathrm{~mm}$ Babcock forceps, and the $10 \mathrm{~mm}$ vessel sealing device were inserted through the SILS port. The left ovary and uterine tube were 

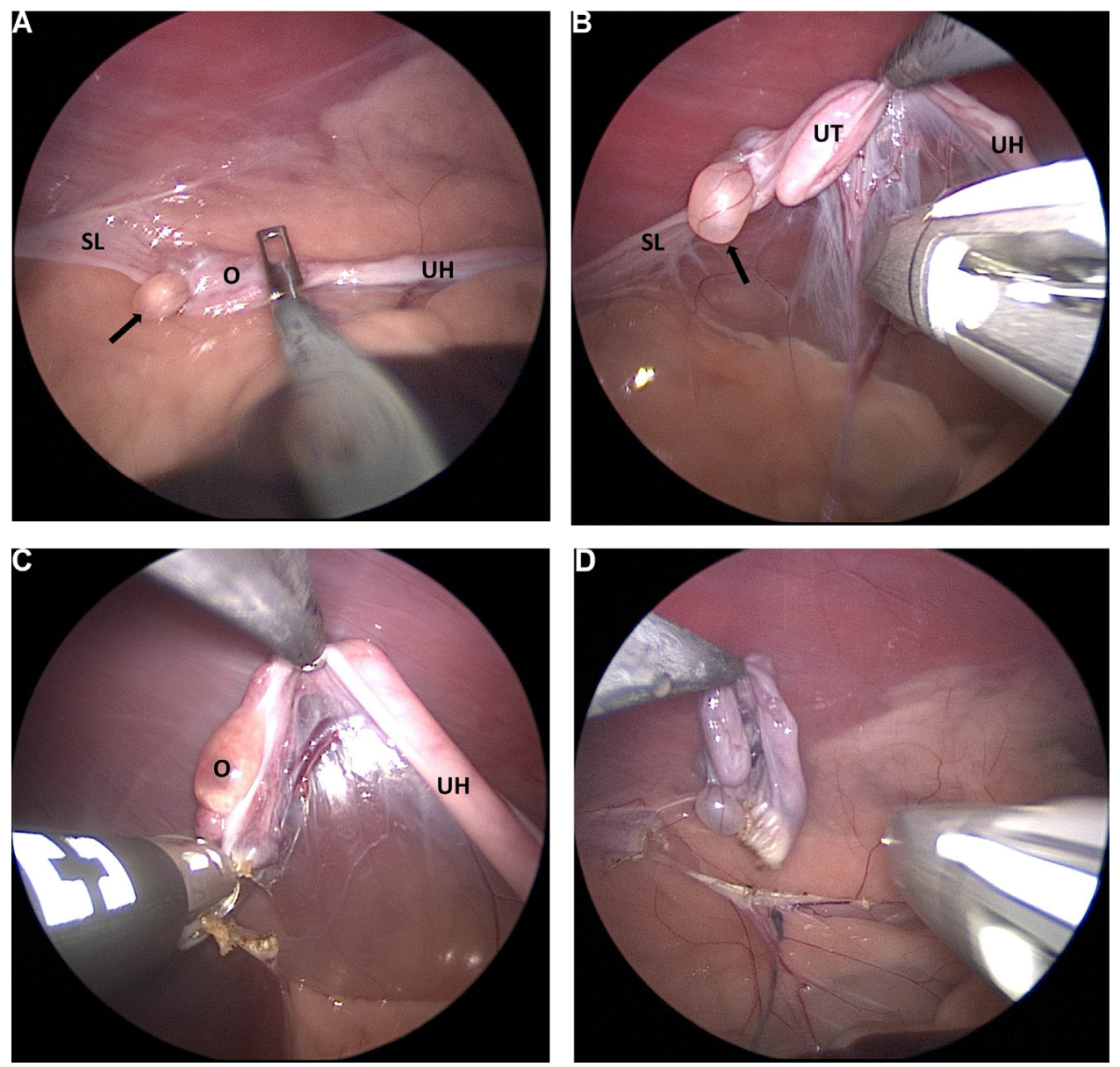

Figure 1 Left ovariectomy in a cheetah. (A) The proper ligament is grasped with a Babcock forceps; (B) The ovary is elevated and the ovarian pedicle is coagulated and transected; (C) The suspensory ligament is coagulated and transected; (D) Ovariectomy is complete with the ovary free from all attachments. Suspensory Ligament (SL), Ovary (O), Uterine Horn (UH), Uterine Tube (UT), Paraovarian cyst (arrow).

visualized. In some instances it was necessary to rotate the ovary in order to access the uterine tube (oviduct or fallopian tube), which was then grasped with the Babcock forceps and elevated slightly (Fig 2A). Using the $10 \mathrm{~mm}$ vessel sealing device, the uterine tube and mesosalpinx at the uterine end of the tube were sealed and transected to perform a distal salpingectomy (Fig 2B). Care was taken to coagulate and cut to the level of the mesovarial margin of the ovary (Video clip 1). The mesosalpinx and severed ends of the uterine tube were observed for hemorrhage and to verify complete transection (Fig 2C). The cheetah was then tilted $45^{\circ}$ into the right oblique position and left salpingectomy was performed as described above.

Once bilateral ovariectomy or salpingectomy was complete, the cheetah was tilted back to the neutral position, the SILS port was removed, and the abdominal cavity deflated. The external rectus fascia was sutured with 0 polydioxanone (PDS Ethicon, Johnson \& Johnson Medical Pty Ltd) using three cruciate sutures. The subcutaneous tissue was closed with 3/0 poliglecaprone 25 (Monocryl Ethicon, Johnson \& Johnson Medical (Pty) Ltd) in a simple continuous pattern and the skin closed with $4 / 0$ poliglecaprone 25 in an intradermal pattern. The surgery duration, the volume of $\mathrm{CO}_{2}$ required to initially achieve $13 \mathrm{mmHg}$, and total volume of $\mathrm{CO}_{2}$ used for each procedure were recorded.

The cheetah was weaned off the ventilator and placed in a transporting crate, extubated, and allowed to recover under constant supervision of the anesthetic team and care takers. The cheetah was monitored intensively during the first 24 hours for any abnormalities in appetite or habitus and for wound dehiscence or hemorrhage. The cheetahs were also frequently observed during the following months.

Re-used SILS ports were disassembled and chemically sterilized for 20 minutes using ortho-phthaldaldehyde (Cidex ${ }^{\circledR}$ OPA, Johnson \& Johnson Medical Pty Ltd), after which they were rinsed in sterile water and dried with a sterile drape.

\section{Statistical Analysis}

The Kolmogorov-Smirnov (KS) test was used to test body weight, age, urine specific gravity, packed cell volume, surgery 

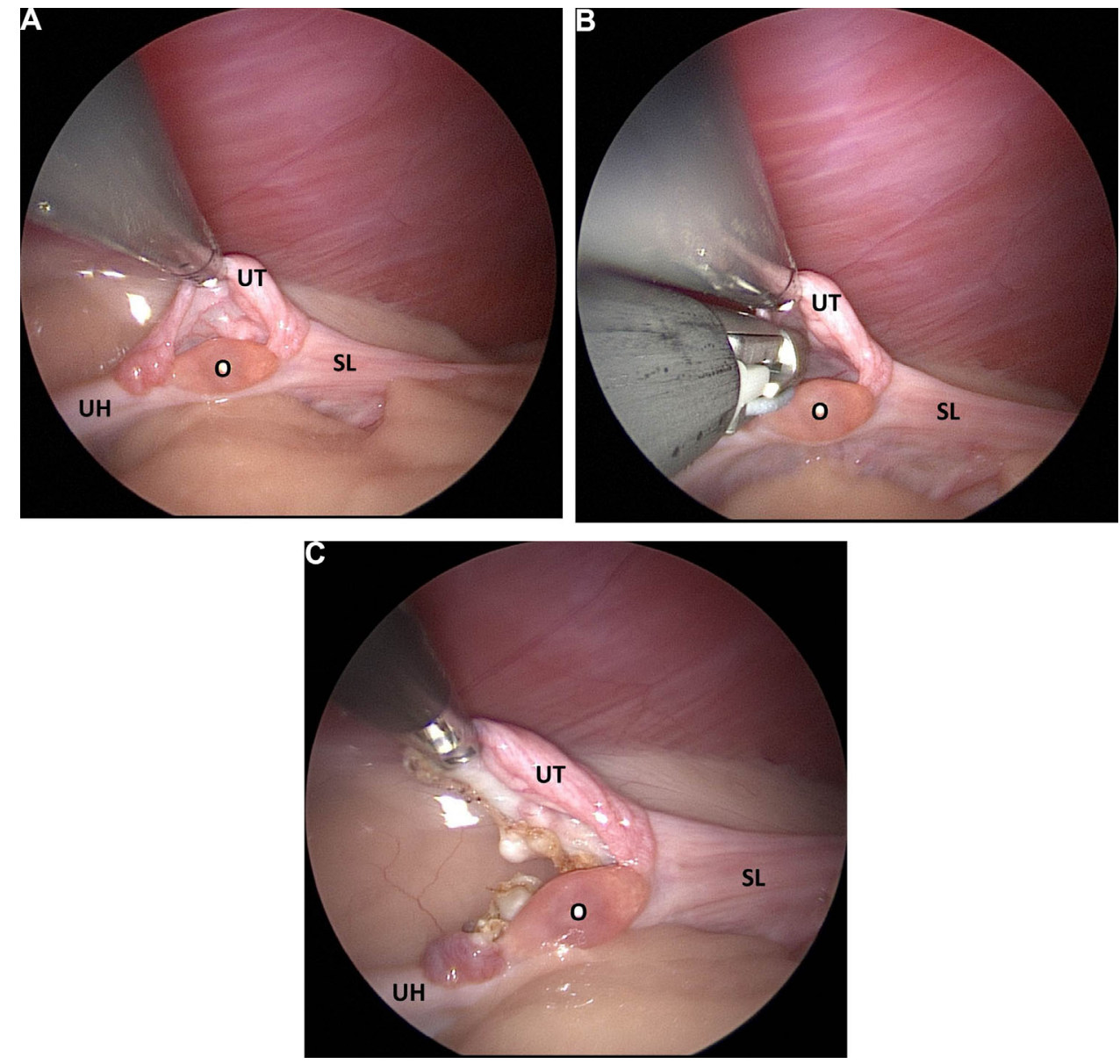

Figure 2 Right salpingectomy in a cheetah. (A) The uterine tube is elevated slightly; (B) The uterine tube and mesosalpinx is coagulated and transected at its uterine end; (C) Salpingectomy complete. Suspensory Ligament (SL), Ovary (O), Uterine Horn (UH), Uterine Tube (UT).

duration and $\mathrm{CO}_{2}$ volume for normality. Normal data were summarized as mean and standard deviation and non-normal data as median and interquartile range (IQR). Data for cheetahs receiving ovariectomy versus salpingectomy were compared using an independent Student's t-test for normal data including body weight $(P=.200, \mathrm{KS})$, urine specific gravity $(P=.119$, $\mathrm{KS})$, and packed cell volume $(P=.200, \mathrm{KS})$. Levene's test for equal variances was used. Data were compared using independent samples median test for non-normal data including age $(P=.034, \mathrm{KS})$, surgery duration $(P=.005)$, volume of $\mathrm{CO}_{2}$ to $13 \mathrm{mmHg}(P=.001)$, and total volume of $\mathrm{CO}_{2}(P=.003)$. All analyses were performed with SPSS v.17 (IBM, Armonk, NY) statistical software. Significance was set at $P<.05$.

\section{RESULTS}

No individuals were excluded from the study due to pregnancy, uterine pathology noted on ultrasound examination, morbid obesity, body condition score of $<2$, or evidence of renal insufficiency (urine specific gravity between 1.008 and 1.015). Data from 1 cheetah that underwent ovariectomy were excluded from the statistical analysis due to surgical complications unrelated to the procedure (an encapsulated foreign body in the abdominal cavity), leaving a total of 20 cheetahs ( $n=10$ for each procedure) in the study.

Age was not significantly different between cheetahs receiving ovariectomy (median 11.5 years, IQR 5.3) versus salpingectomy (median 11.5 years, IQR 4.9) $(P=1.0)$. The body weight was not significantly different between cheetahs receiving ovariectomy (mean $\pm \mathrm{SD}, 32.6 \pm 2.0 \mathrm{~kg}$ ) and salpingectomy $(35.8 \pm 3.6 \mathrm{~kg})(P=.571)$. The mean $\pm \mathrm{SD}$ urine specific gravity for the entire cohort was $1.05 \pm 0.008$ and packed cell volume was $46 \pm 3 \mathrm{~L} / \mathrm{L}$.

The fat content in the subcutis, falciform, and median urinary bladder ligament and ovarian pedicle was minimal. Two applications of the vessel sealing device were required to successfully coagulate and transect all uterine tubes and uterine horns. All procedures were successfully completed 
through the SILS port using straight instruments. The endoscope with the light post at the top was placed in the $5 \mathrm{~mm}$ cannula at the 12 o'clock position in the SILS port, the grasping forceps in the $5 \mathrm{~mm}$ cannula at 8 o'clock and the vessel sealing device in the $5-12 \mathrm{~mm}$ cannula at 4 o'clock. Half of the SILS ports were re-used. Specific placement of the laparoscopic instruments facilitated the procedure. None of the cheetahs required enlargement of the portal incision to retrieve the ovaries. Paraovarian cysts were identified in 24 of 42 ovaries at laparoscopy. The linea alba was clearly identified and incised in half the cheetahs and a paramedian incision was performed in the other half. No intraoperative complications occurred and none of the animals developed subcutaneous emphysema during or immediately after surgery. Contraction of the suspensory ligament and mesosalpinx was observed in some cheetahs.

An intra-abdominal mass was identified by transabdominal ultrasound preoperatively in 1 cheetah and fine needle aspiration of the mass showed moderate numbers of neutrophils, macrophages, and a mixed population of bacteria. Ovariectomy was performed after laparoscopic removal of the mass. The mass was located within the omentum and was not attached to any abdominal organ. The vessel sealing device was used to free the mass from the omentum and a retrieval bag was used to remove the excised mass. The mass was approximately $6 \mathrm{~cm}$ in diameter and required extension of the portal incision for retrieval. A thorn foreign body was subsequently identified in the center of the mass. This cheetah was excluded from data analysis in the study.

The surgery duration for ovariectomy (median 24 minutes, IQR 3) was significantly longer than for salpingectomy (median 19.5 minutes, IQR 3) $(P=.005)$. The volume of $\mathrm{CO}_{2}$ used to insufflate the peritoneal cavity to $13 \mathrm{mmHg}$ was not significantly different for cheetahs receiving ovariectomy (median 3.25 L, IQR 0.45) or salpingectomy (median 3.25 L, IQR 0.52) $(P=1.0)$. The total volume of $\mathrm{CO}_{2}$ required was significantly higher for cheetahs receiving ovariectomy (median $11.25 \mathrm{~L}$, IQR 3.08) than salpingectomy (median 4.90 L, IQR 2.52) $(P=.001)$. Note, this included data from 1 cheetah that had some $\mathrm{CO}_{2}$ valve leakage throughout salpingectomy.

All cheetahs returned to normal behavior and appetite within 18 hours of surgery. One cheetah self-mutilated its tail and a leg in the recovery period but stopped once it was fully recovered. One cheetah developed visible signs of mild inflammation around the portal incision that resolved within 24 hours. At facility B, none of the cheetahs developed incisional hernias. The frequency of incisional hernias at facility A was unknown as the cheetahs were not tame; however, no incidents were reported by the caretakers. No surgically related complications were reported for any cheetah 3 months after surgery.

\section{DISCUSSION}

Laparoscopic ovariectomy and salpingectomy can be performed in cheetahs using a SILS port without intraoperative complications. The lean physiology of cheetahs provides optimal conditions for laparoscopic surgery. Most cheetahs had minimal subcutaneous and intra-abdominal fat in the median and falciform ligaments compared to that encountered in the adult lioness, but the fat distribution was similar to subadults lions or cubs. ${ }^{18}$ Similar to the lion, minimal fat was present in the ovarian pedicle and it did not vary markedly between individuals as is reported in dogs. The uterine horns were small and coagulation of the proper ligament and tip of the uterine horns was relatively easy, requiring only 2 applications of the vessel sealing device. In lions, more applications were required. ${ }^{18}$ Distal salpingectomy was performed similar to that in women. ${ }^{29,30}$ This was done with 2 applications of the vessel sealing device comparable to findings in the lion. ${ }^{18}$ Similar to the lion, neither the Trendelenburg position ${ }^{9,11,31}$ nor a true lateral position ${ }^{23}$ were required to access ovaries in the cheetahs although some manipulation of the spleen was required to access the left ovary.

A SILS port was used to reduce the number of incisions and portals, which can reduce surgery duration and limit the risk of inadvertent trauma to visceral organs. It must be noted that the risk of herniation, however, might be higher for a $2 \mathrm{~cm}$ incision compared to 2 or 3 smaller incisions used for a multiport technique. Three ports were necessary for salpingectomy. The SILS port eliminated the need for transcutaneous suspension of the ovary for ovariectomy since 1 port could be used for grasping forceps. ${ }^{13,34}$ The SILS port obviates the need for Veress needle insertion ${ }^{18,32}$ or a modified Hasson technique, ${ }^{18,33}$ which simplified the approach.

Ovariectomy with laparoscopy has been described with 1-, 2-, and 3-ports in dogs and cats, ${ }^{13,21-24}$ and a 3-port technique that allows for triangulation has been previously described in lions. ${ }^{18}$ The 2-port technique with percutaneous transfixation of the ovary appeared to provide the best compromise between surgery duration and pain after surgery. ${ }^{22,24,35}$ However, it required a transcutaneous suspension of the ovary, impossible for the salpingectomy procedure. The 1-port technique could not be used as a $10 \mathrm{~mm}$ vessel sealing device was a prerequisite for sealing the uterine horn ${ }^{36}$ and similarly, salpingectomy would also not have been possible.

Curved or articulated instruments have been designed for the SILS port to prevent crowding of the instrumentation. ${ }^{37}$ In this study we used straight instruments, placed and positioned as described earlier, with minimal interference encountered between instrument handles or tips. In all cheetahs, access to the ovaries was easily achieved with $36 \mathrm{~cm}$ instruments as is the experience in lions. ${ }^{18}$ The SILS ports may be re-used; ${ }^{28}$ however, damage to the valve in the cannulas can result in $\mathrm{CO}_{2}$ leakage.

The SILS port required a $2 \mathrm{~cm}$ incision on ventral midline incision. Removal of the SILS after each ovariectomy facilitated the retrieval of each ovary. It was unnecessary to enlarge the portal incision as is the case with large ovaries. Since the portal incision was not enlarged for retrieval of the 1st ovary, a good seal was still achieved with replacement of the SILS port for the 2nd ovariectomy. In a previous study on lions, ovaries could be retrieved through the $12 \mathrm{~mm}$ cannula in sub-adult lionesses but the $12 \mathrm{~mm}$ cannula had to be removed with the ovaries in adult lions. ${ }^{18}$ In the current study, 
retrieval of the ovaries in some cheetahs would have been possible via the 5-12 mm cannula of the SILS port. However, the prevalence of paraovarian cysts in more than half the cheetahs in this study was much higher compared to that in lions, ${ }^{38}$ and the tissue bulk or the risk of the cysts bursting made removal of the ovary through the cannula less favorable, thus the SILS port was removed for the retrieval of all ovaries.

No external signs of gross uterine pathology were observed in any cheetahs in this cohort of older, barren, nulliparous cheetahs that had not been bred due to legislative restrictions. In wild canids captive in North American zoos, the number of years the animal has been barren has been implied to be associated with the occurrence of uterine pathology $^{39}$ and it was thus interesting not to observe any pathology in the current study.

No intraoperative complications were encountered during either procedure. Identification of the linea alba was not always possible compared to the lioness, where it was constantly found, necessitating a paramedian approach in some cheetahs. ${ }^{18}$ The SILS was placed successfully with a tight seal in every cheetah and no obvious $\mathrm{CO}_{2}$ leakage (except for 1 valve leakage) or subcutaneous emphysema was encountered during or after surgery. The vessel sealing device achieved excellent hemostasis in all cheetahs. None of the uterine horns failed to seal with the vessel sealing device at the time of surgery. This is consistent with reports that a $10 \mathrm{~mm}$ vessel sealing device can safely seal a $9 \mathrm{~mm}$ canine uterine horn. ${ }^{36}$

Physical contraction of the suspensory ligament in the cheetah was observed (Video clip 2) similar to that reported in the lioness. ${ }^{40}$ Contraction of the mesosalpinx was also noted in 1 cheetah. These contractions seemed to be induced by physical manipulation and prompts further investigation of the histologic composition and the functionality of the suspensory and broad ligaments.

Laparoscopic salpingectomy in the cheetah is faster than ovariectomy using SILS. The $\mathrm{CO}_{2}$ volume required to insufflate the peritoneal cavity to a pressure of $13 \mathrm{mmHg}$ was constant in all cheetahs as expected with consistent body size. However, significantly more $\mathrm{CO}_{2}$ was required to complete ovariectomy compared to salpingectomy. Removal of the SILS port to retrieve the 1st ovary, which then required a re-insufflation of the peritoneal cavity, was the main reason for using more $\mathrm{CO}_{2}$ during ovariectomy. Sterilization of wild felids typically occurs in remote areas and pre-determined consumable requirements assist in accurate planning of these projects.

Salpingectomy, opposed to ovariectomy, was considered in this species to avoid the known disadvantages of gonadectomy. ${ }^{41}$ Bilateral salpingectomy in women is currently preferred over ovariectomy since ovariectomy is associated with more long-term negative effects..$^{20,30,42}$ The potential for development of pyometra subsequent to long-term uterine exposure to sex steroids in cheetahs seems to be minimal. ${ }^{43,44}$ The advantages and disadvantages of gonadectomy in animals $^{41}$ have to be considered prior to surgical sterilization of wild African felids, and the long-term risks of salpingectomy are not known.

We showed that laparoscopic ovariectomy and salpingectomy was safely and successfully performed in cheetahs.
The use of SILS allowed easy access to the peritoneal cavity. The poorly-developed mesosalpinx and ovarian bursa in cheetahs, similar to the lioness, facilitated access to the uterine tube for salpingectomy. ${ }^{18}$

\section{ACKNOWLEDGMENTS}

This study was partly funded by NRF grants of Professor Schoeman and Professor Kirberger. The authors acknowledge Karl Storz (South Africa and Namibia) for supplying and managing all laparoscopic equipment and instrumentation, Karl Storz (Germany) and AfriCat for funding transport, Covidien (USA) for supplying SILS ports and Covidien (South Africa) for supplying LigaSure hand pieces. The authors acknowledge the assistance of Dr. Adrian Tordiffe for the anesthetic management of the cheetahs and the Department of Companion Animal Clinical Studies, Faculty of Veterinary Science, University of Pretoria, Pretoria, South Africa, and the Arabella Dean fund of the South African Veterinary Foundation for funding. The authors thank the owners, directors, and staff of AfriCat and Cheetah Conservation Foundation for facilitating the study.

\section{DISCLOSURE}

The authors declare no conflicts of interest related to this report.

\section{REFERENCES}

1. Jago M: Ministry of Environment and Tourism, Republic of Namibia: numbers of cheetah in captivity. 2014

2. Ministry of Environment and Tourism, Republic of Namibia: regulations for large carnivores in captivity: Nature conservation ordinance 1975. Government Gazette 2012;4911

3. Bertschinger $\mathrm{H}$, Trigg $\mathrm{T}$, Jochle $\mathrm{W}$, et al: Induction of contraception in some african wild carnivores by downregulation of LH and FSH secretion using the GnRH analogue deslorelin. Fertility control in wildlife. 2002;41-52

4. Bertschinger $\mathrm{H}$, Jago M, Nothling J, et al: Repeated use of the $\mathrm{GnRH}$ analogue deslorelin to down-regulate reproduction in male cheetahs (Acinonyx jubatus). Theriogenology 2006;66:1762-1767

5. Okkens A, Kooistra H, Nickel R: Comparison of long-term effects of ovariectomy versus ovariohysterectomy in bitches vergleich der langzeiteffekte der ovarektomie mit denen der ovariohysterektomie bei der hundin. Prakt Tierarzt 2003;84:98-101

6. Howe LM: Surgical methods of contraception and sterilization. Theriogenology 2006;66:500-509

7. Goethem Bv, Schaefers-Okkens A, Kirpensteijn J: Making a rational choice between ovariectomy and ovariohysterectomy in the dog: a discussion of the benefits of either technique. Vet Surg 2006;35:136-143

8. DeTora M, McCarthy R: Ovariohysterectomy versus ovariectomy for elective sterilization of female dogs and cats: is removal of the uterus necessary? J Am Vet Med Assoc 2011;239:1409-1412 
9. Austin B, Lanz OI, Hamilton S, et al: Laparoscopic ovariohysterectomy in nine dogs. J Am Anim Hosp Assoc 2003;39:391-396

10. Davidson E, Moll H, Payton M: Comparison of laparoscopic ovariohysterectomy and ovariohysterectomy in dogs. Vet Surg 2004;33:464-470

11. Hancock R, Lanz OI, Waldron D, et al: Comparison of postoperative pain after ovariohysterectomy by harmonic scalpelassisted laparoscopy compared with median celiotomy and ligation in dogs. Vet Surg 2005;34:273-282

12. Wildt DE, Lawyer DF: Laparoscopic sterilization of the bitch and queen by uterine horn occlusion. Am J Vet Res 1985;46:864-869

13. Devitt CM, Cox RE, Hailey JJ: Duration, complications, stress, and pain of open ovariohysterectomy versus a simple method of laparoscopic-assisted ovariohysterectomy in dogs. $J$ Am Vet Med Assoc 2005;227:921-927

14. Nimwegen SAv, Kirpensteijn J: Laparoscopic ovariectomy in cats: comparison of laser and bipolar electrocoagulation. J Feline Med Surg 2007;9:397-403

15. Hartman MJ, Kirberger RM, Tordiffe ASW, et al: Laparoscopic removal of a large abdominal foreign body granuloma using single incision laparoscopic surgery (SILS) and extraction bag in a cheetah (Acinonyx jubatus). Vet Rec Case Rep 2015;3: DOI:10.1136/vetreccr-2014-000162

16. Aguilar RF, Mikota SK, Smith J, et al: Endoscopic ovariohysterectomy in two lions (Panthera leo). J Zoo Wildl Med 1997;28:290-297

17. Kolata RLaparoscopic ovariohysterectomy and hysterectomy on African lions (Panthera leo) using the UltraCision harmonic scalpel. 2 J Zoo Wildl Med 2002;33:280-282

18. Hartman MJ, Monnet E, Kirberger RM, et al: Laparoscopic sterilization of the African lioness (Panthera leo). Vet Surg 2013;42:559-564

19. Emerson JA, Case JB, Brock AP, et al: Single-incision, multicannulated, laparoscopic ovariectomy in two tigers (Panthera tigris). Vet Q 2013;33:108-111

20. Parker W:Oophorectomy versus salpingectomy: a converge of ideas. Menopause 2014;21:323-324

21. Runge JJ, Curcillo PG II, King SA, et al: Initial application of reduced port surgery using the single port access technique for laparoscopic canine ovariectomy. Vet Surg 2012;41:803-806

22. Coisman JG, Case JB, Shih A, et al: Comparison of surgical variables in cats undergoing single-incision laparoscopic ovariectomy using a LigaSure or extracorporeal suture versus open ovariectomy. Vet Surg 2014;43:38-44

23. Dupre G, Fiorbianco V, Skalicky M, et al: Laparoscopic ovariectomy in dogs: comparison between single portal and twoportal access. Vet Surg 2009;38:818-833

24. Case JB, Marvel SJ, Boscan P, et al: Surgical time and severity of postoperative pain in dogs undergoing laparoscopic ovariectomy with one, two, or three instrument cannulas. J Am Vet Med Assoc 2011;239:203-208

25. Manassero M, Leperlier D, Vallefuoco R, et al: Laparoscopic ovariectomy in dogs using a single-port multiple-access device. Vet Rec 2012

26. Wilson D, Monnet E: The use of single incision laparoscopic surgery (SILS Port) in dogs: description of the technique and initial impressions after 22 consecutive cases. Proceedings of the 9th Annual Meeting of the Veterinary Endoscopic Society, Park City, UT: 2012; pp. 22-24

27. Case JB, Ellison G: Single incision laparoscopic-assisted intestinal surgery (SILAIS) in 7 dogs and 1 cat. Vet Surg 2013;42:629-634

28. Coisman JG, Case JB, Clark ND, et al: Efficacy of decontamination and sterilization of a single-use single-incision laparoscopic surgery port. Am J Vet Res 2013;74:934-938

29. Miller DM, McAlpine JN, Gilks CB, et al: Opportunistic salpingectomy: the way forward-response to Steven Narod. Curr Oncol 2013;20:143-144

30. Hseih G, Antony K, Masand R, et al: A prospective feasibility study of postpartum distal salpingectomy. Obstet Gynecol 2014;123:92S

31. Goethem BEBJv, Rosenveldt KW, Kirpensteijn KW: Monopolar vs. bipolar electrocoagulation in canine laparoscopic ovariectomy: a nonrandomized, prospective, clinical trial. Vet Surg 2003;32:464-470

32. Monnet E, Twedt D: Laparoscopy. Vet Clin North Am Small Anim Pract 2003;33:1147-1163

33. Hasson AA modified instrument and method for laparoscopy. Am J Obstet Gynecol 1971;110:886-887

34. Laparoscopic spay in dogs and cats [DVD]. Karl Storz, GMBH C \& Co KG, editor. Tuttlingen, Germany, 2006

35. Boscan P, Monnet E, Mama K, et al: A dog model to study ovary, ovarian ligament and visceral pain. Vet Anaesth Analg 2011;38:260-266

36. Barrera JS, Monnet E: Effectiveness of a bipolar vessel sealant device for sealing uterine horns and bodies from dogs. Am J Vet Res 2012;73:302-305

37. Runge JJ, Mayhew PD: Evaluation of single port access gastropexy and ovariectomy using articulating instruments and angled telescopes in dogs. Vet Surg 2013;42:807-813

38. Kirberger R, Schulman M, Hartman M: Ultrasonographic and laparoscopic evaluation of the reproductive tract of the captive female African lion (Panthera leo). Theriogenology 2011;76:810-818

39. Asa CS, Bauman KL, Devery S, et al: Factors associated with uterine endometrial hyperplasia and pyometra in wild canids: Implications for fertility. Zoo Biol 2014;33:8-19

40. Hartman M, Groenewald H, Kirberber R: Morphology of the female reproductive tract of the African lion (Panthera leo). Acta Zoologica 2013;94:437-446

41. Kustritz MVR:Effects of surgical sterilization on canine and feline health and on society. Reprod Dom Anim 2012;47:214-222

42. Backes FJ:Salpingectomy, why not? Am J Obstet Gynecol 2014;211:385-386

43. Crosier A, Comizzoli P, Baker T, et al: Increasing age influences uterine integrity, but not ovarian function or oocyte quality, in the cheetah (Acinonyx jubatus). Biol Reprod 2011;85:243-253

44. Wachter B, Thalwitzer S, Hofer H, et al: Reproductive history and absence of predators are important determinants of reproductive fitness: the cheetah controversy revisited. Conservation Letters 2011;4:47-54 